\title{
A Model Ground State of Polyampholytes
}

\author{
Shay Wolfling and Yacov Kantor \\ School of Physics and Astronomy, Tel Aviv University, Tel Aviv 69978, Israel
}

\begin{abstract}
The ground state of randomly charged polyampholytes is conjectured to have a structure similar to a necklace, made of weakly charged parts of the chain, compacting into globules, connected by highly charged stretched 'strings'. We suggest a specific structure, within the necklace model, where all the neutral parts of the chain compact into globules: The longest neutral segment compacts into a globule; in the remaining part of the chain, the longest neutral segment (the 2nd longest neutral segment) compacts into a globule, then the 3rd, and so on. We investigate the size distributions of the longest neutral segments in random charge sequences, using analytical and Monte Carlo methods. We show that the length of the $n$th longest neutral segment in a sequence of $N$ monomers is proportional to $N / n^{2}$, while the mean number of neutral segments increases as $\sqrt{N}$. The polyampholyte in the ground state within our model is found to have an average linear size proportional to $\sqrt{N}$, and an average surface area proportional to $N^{2 / 3}$.
\end{abstract}

36.20.-r,02.50.-r,05.40.+j,33.15.Bh

\section{INTRODUCTION}

The desire to understand long chain biological macromolecules, and especially proteins, stimulates extensive studies of polymers [1] 3]. An important class of polymers are those with an electric charge along their backbone. This work deals with heteropolymers, which carry positive and negative charges, known as polyampholytes (PA's) [4. Models of PA's are important to the study of proteins, since under normal physiological conditions, 5 of the 20 naturally occurring amino acids have an excess charge [1]. We consider a polymer of charged monomers, interacting via unscreened Coulomb interactions, and we investigate its ground state structure. Throughout this work, we discuss PA's that consist of a random mixture of positive and negative charges, which cannot move along the chain.

We are interested in the geometrical features of the ground state of a PA, and in particular in the dependence of its radius of gyration (r.m.s. size) $R_{g}$ on the number of monomers $N: R_{g} \sim N^{\nu}$. Higgs and Joanny [5] and later Wittmer et al. [6], elaborated on arguments of Edwards et al. [7] and suggested, on the basis of a DebyeHückel type theory [8], a collapsed structure $(\nu=1 / 3)$ for neutral PA's in the ground state: The chain takes advantage of the presence of two types of charges along its backbone, and assumes a spatial conformation in which every charge is predominantly surrounded by charges of an opposite sign.

A different approach to the study of the ground state of PA's [9], is by scaling arguments, requiring that the interaction energy be the same on all length scales. These arguments lead to a stretched structure of the ground state, where $R_{g} \sim N$. In this approach $R_{g}$ is averaged over a complete ensemble of all quenches, and the typical overall (excess) charge $Q$ of the PA is $\sim \sqrt{N}$. Whereas in the Debye-Hückel approximation, all the chains are neutral, since the overall neutrality of the PA is an essential condition for the validity of the Debye-Hückel approx- imation. The extreme sensitivity of the ground state structure to the excess charge, noted by Kantor et al. [10,11, and supported by Monte Carlo (MC) simulations as well as variational mean field calculations [12], resolves the apparent contradiction between the scaling and the Debye-Hückel motivated arguments.

To gain some insight into the behavior of PA's, analogies to charged drops were explored [13 15]: A spherical drop charged beyond a certain charge, called the Rayleigh charge $Q_{R}$, which depends on the surface tension and volume of the drop, becomes locally unstable to elongation, since the pressure difference between the inside and outside of the drop vanishes. Even before the total charge reaches $Q_{R}$, the drop becomes unstable to splitting into two equal drops. Additional splittings of the drop occur for larger charges. Similar behavior is expected in PA's charged to $Q_{R} \sim \sqrt{N}$. Although a PA cannot split, the analogy to charged drops can still be exploited: Constraining the structure to maintain its connectivity by attaching droplets with narrow tubes, results in a necklace-type structure of droplets connected by strings.

For homogeneously charged polymers (polyelectrolytes), the charged drop analogy was used [16] to characterize the structure completely within the necklace model (including the number of 'beads' and strings, their sizes, and the number of monomers in them, for a given temperature and charge density). However, trying to apply the necklace model to quenched PA's having random charges [13,14], several difficulties occur due to the randomness. It was noted, for instance, that a situation occurs, in which most spherical shapes are unstable, while there is on average no energetic gain in splitting the sphere into two equal parts. A consistent theoretical picture for random PA's beyond the instability threshold was not found, but a typical PA is conjectured to be composed of rather compact globules connected by long strings. In order to reduce the electrostatical energy, the globules consist of segments of the chain that are approxi- 
mately neutral (collapsing according to the Debye-Hückel picture), while the strings are formed by highly charged segments.

Since the structure of a randomly charged PA cannot be characterized completely analytically, we turn to numerical MC methods in order to characterize the ground state of such PA's within the necklace model. A key role in the structure of randomly charged PA's is played by the neutral segments in the chain (forming the beads in the necklace). We therefore apply MC methods to study the neutral segments size distribution of randomly charged PA's. The rest of the paper is organized as follows: In section II we describe the process of construction of a ground state for a randomly charged PA, by dividing it into neutral segments. We discuss the motivation for this process, define the important parameters of the problem and compare the process to similar existing models. In section III we investigate the sizes of the globules in the suggested ground state, including their dependence on the total number of monomers and on other parameters. In section IV we discuss effects of finite chains, investigate the dependence of the total number of neutral segments in such finite chains on $N$, and construct a self consistent picture of the structure of the ground state. In section $\square$ we obtain some of the physical characteristics of the constructed ground state, such as its linear size and surface area, and in the last section we discuss our results and compare them to other studies.

\section{THE MODEL - MOTIVATION AND DEFINITIONS}

Empirical observations of the energy of randomly charged PA's suggested that the quench-averaged energy can be presented as a sum of condensation, surface and electrostatic energies [13,14]:

$$
E=-\frac{q_{0}^{2}}{a} N+\gamma S+\frac{Q^{2}}{R}
$$

where $q_{0}^{2} / a$ is the condensation energy gain per particle $\left(q_{0}\right.$ is the typical charge of a monomer and $a$ is a microscopic distance such as diameter of the monomer), $\gamma \sim q_{0}^{2} / a^{3}$ is the surface tension, and $R$ is the linear size of the chain. (In Eq. (11) we omitted dimensionless prefactors of order unity.) When the excess charge $Q$ is very small, the PA will form a single globule. When $Q$ increases, the electrostatic forces will overcome the surface tension and the globule will split (similarly to the splitting of a drop charged beyond the Rayleigh charge). Numerical studies suggest [13 15] that a PA forms a necklace of weakly charged globules, connected by highly charged strings. This structure is a compromise between the tendency to reduce the surface area (i.e. to form globules) due to surface tension, and the tendency to expand, in order to reduce the Coulomb interaction caused by the excess charge.
Kantor and Ertas 17 19] attempted to quantify the qualitative necklace model, by postulating that the ground state of a PA will consist of a single globule, formed by the longest neutral segment of the PA, while the remaining part will form a tail. They investigated the probability that the longest neutral segment in a chain of $N$ monomers has length $L$. A probability density was defined and investigated in the limit where $N, L \rightarrow \infty$, while the reduced length $l \equiv L / N$ is fixed. The probability density was obtained numerically and investigated analytically, but a complete analytical solution to the problem was not found. We follow [17], and investigate the problem of the size distribution of neutral segments in randomly charged PA's by mapping the charge sequence into a one-dimensional random walk (1-d RW). The charge sequence $\omega=\left\{q_{i}\right\}\left(i=1, \ldots, N ; q_{i}= \pm 1\right)$ is mapped into a sequence of positions $S_{i}(\omega)=\sum_{j=1}^{i} q_{j}$ $\left(S_{0}=0\right)$ of a random walker. (From now we will measure charges in units of the basic charge $q_{0}$, and therefore $q_{j}$ will be dimensionless.) The random sequence of charges is thus equivalent to a RW, a chain segment with an excess charge $Q$ corresponds to a RW segment with total displacement of $Q$ steps, and a neutral segment is equivalent to a loop inside the RW. Throughout the paper we will use the terminologies of randomly charged PA's and of RW's interchangeably.
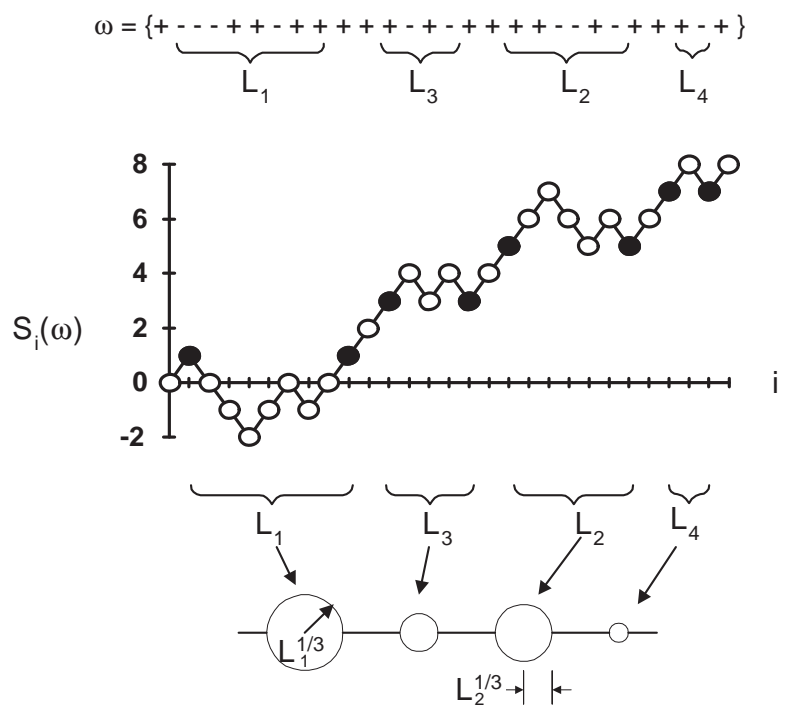

FIG. 1. An example of a charge sequence $\omega$, mapped into a 1-d RW $S_{i}(\omega)$, and a typical loops structure. Filled circles indicate the starting and ending points of loops. The longest loop in the RW has 8 steps $\left(L_{1}=8\right)$, the 2nd longest loop has 6 steps $\left(L_{2}=6\right)$, $L_{3}=4$ and $L_{4}=2$. The excess charge (which is equivalent to the total displacement of the RW) is $Q=+8$ and the total length is $N=28$.

When the longest neutral segment forms a globule (as assumed in [17 [19]), the remaining part of the chain is very large $(\sim N)$. It is natural to assume that neutral segments on that tail will further reduce the total energy by folding into globules. Eventually, the necklace 
will consist of many neutral globules. However, there are many ways in which the chain can be divided into neutral segments, and we are interested in a simple unique structure. We therefore suggest a specific necklace-type structure, and construct the ground state for a randomly charged PA in the following way, depicted in Fig. 1ㅑ The longest neutral segment contains $L_{1}$ monomers; it compacts into a globule of linear size proportional to $L_{1}^{1 / 3}$; in the remaining part of the chain the longest neutral segment (the 2nd longest neutral segment) of size $L_{2}$ also compacts into a globule, then the 3rd and so on, until the segments become very small (of only a few monomers). Generally, $L_{n}$ denotes the number of monomers in the $n$th longest neutral segment, which compacts into a globule of radius $L_{n}^{1 / 3}$. Eventually, all the neutral segments are exhausted and we are left only with strings which carry the PA's excess charge $Q$, and connect the globules. The total number of monomers in the chain is the number of monomers in all the neutral segments, plus the excess charge.

The 'ground state', generated by this process of longest neutral segments compacting into globules, does not necessarily minimize the total energy of the PA. First of all, the process does not consider the possibility of weakly charged globules, which can include much more monomers than the neutral globules, thus compensating by surface energy for the additional electrostatic energy. Secondly, even when considering only neutral globules, it is not necessary that the procedure of compacting the longest neutral segment at each step generates the lowest energy state: It is possible that two 'medium sized' globules will have lower surface energy than a long one and a very short one (which remains after the first long segment was already chosen).

A similar model, describing a breaking of a RW into loops, is the Loop Erased Self-Avoiding Walk (LESAW) model [20]. A LESAW is constructed from a RW, in which any loop generated by a self intersection is erased. The LESAW model was subject for intensive study 21 25], and many properties of the lengths of the erased loops in it were found. However, these results do not consider issues of the longest erased loops, and are therefore of little help to us.

Other related models are those of randomly broken objects 26,27, in which an object, such as a segment of unit length, is divided into mutually exclusive parts by a self-similar random process. The division of a segment into parts resembles the 'breaking' of a RW into loops: The probability densities of the longest segments in some models of randomly broken objects resemble the probability density of the longest loop in [18]. However, one main difference between models of randomly broken objects and the longest loop problem, is that the probabilities of the longest loops are not self-similar. (The probability of a certain fraction of the chain to form the longest loop and the probability of the same fraction of the remaining chain to form a second longest loop are dif- ferent.) We will return to this absence of self-similarity in section III].

\section{SIZE DISTRIBUTION OF NEUTRAL SEGMENTS}

In order to characterize a PA in the necklace-type low energy state, made of neutral globules and highly charged strings, we are interested not just in the size distribution of the longest neutral segment, but also in the size distribution of the 2nd longest segment, 3rd longest, and so on. We examined the statistics of loops in a 1-d RW of $N$ steps, using MC method for several $N$ 's, up to $N=10^{4}$. For each $N$ we randomly selected $10^{6}$ sequences. For each sequence we found the lengths $L_{n}$ of the (non-overlapping) loops, and calculated their distributions and averages.

We denote by $P_{N, n}\left(L_{n}\right)$ the probability of the $n$th longest loop in a RW of $N$ steps to be of length $L_{n}$. The average length of the longest loop was found 17,18 to be proportional to $N$. Expecting the same behavior for the length of the $n$th longest loop, we define the probability density of the $n$th longest loop:

$$
p_{n}\left(l_{n}\right) \equiv \frac{N}{2}\left[P_{N, n}\left(L_{n}\right)+P_{N, n}\left(L_{n}+1\right)\right],
$$

where $l_{n} \equiv L_{n} / N$. (Note that at least one term in Eq. (2) vanishes, since loops can be only of even length. Therefore, definition (2) includes average of probabilities for $L_{n}$ and $L_{n}+1$ as in the definitions used in continuum limits for discrete RW's, in order to prevent evenodd oscillations.) For small $N$ 's this definition of $p_{n}\left(l_{n}\right)$ still depends on $N$, but in the $N, L_{n} \rightarrow \infty$ limit it becomes a function of only $l_{n}$. Numerical evidence for the $N$-independence of $p_{1}\left(l_{1}\right)$ for $N \rightarrow \infty$ was presented in [18. MC results for $p_{n}\left(l_{n}\right)$ for several values of $n$ indicate that the probability densities are virtually independent of $N[28$. For small $N$ and large $n$ the probability density $p_{n}\left(l_{n}\right)$ depends on $N$, since $L_{n}$ 's are short, and continuum limit is expected only when $L_{n} \gg 1$. In order to overcome effects of finite $N$, we determined the $N$-dependence of $\left\langle L_{n}\right\rangle$, through an extrapolation of the slopes of the linear fits of $\log \left\langle L_{n}\right\rangle$ vs. $\log N$ to $N \rightarrow \infty$. For any given $n$ we calculated, through a local linear fit, slopes for several values of $N$, and estimated them at $N \rightarrow \infty$ limit. Furthermore, it can be proven analytically 28] that the probability density of the longest loop is 'universal', i.e. for long RW's, it does not depend on the number of steps, or on details of a single step.

Since $p_{n}\left(l_{n}\right)$ are $N$-independent, we can investigate them for any given (large enough) $N$. The probability densities of the five longest loops in a chain are depicted in Fig. 2 for $N=1000$. Several properties of $p_{n}\left(l_{n}\right)$ are evident from this figure. The probability density $p_{1}\left(l_{1}\right)$ was shown 18 to have a square root divergence at $l_{1}=1$, and a discontinuous derivative at $l_{1}=\frac{1}{2}$. When the length of the first loop $L_{1}$ in a specific chain is long, 
then the lengths of the other loops $L_{n}$ (for $n>1$ ) in that chain must be short, since the total length of all the loops cannot exceed $N$. Therefore, since in many chains the length of the longest loop is almost equal to $N$ (as indicated by the divergence of $p_{1}\left(l_{1}\right)$ when $l_{1} \rightarrow 1$ ), the lengths of the other loops approach zero. This is evident from the divergence of $p_{n}\left(l_{n}\right)$ when $l_{n} \rightarrow 0$ for all $n>1$. Because in any specific chain the length of the $n$th longest loop is shorter than the length of the $k$ th longest loop, for $n>k$, the divergence of $p_{n}\left(l_{n}\right)$ near zero is stronger for large $n$. Since the probability densities $p_{n}\left(l_{n}\right)$ are normalized separately for each $n$, then any two of them must intersect (i.e. $p_{n}\left(l_{n}\right)$ always intersects $p_{n^{\prime}}\left(l_{n^{\prime}}\right)$ for $n \neq n^{\prime}$ ). The length of the second longest loop never exceeds the length of the first longest loop, and the sum of their lengths never exceeds $N$. Therefore, the length of the second longest loop cannot exceed half the length of the chain. Consequently, $l_{2} \leq \frac{1}{2}$ for all the chains, and $p_{2}\left(l_{2}\right)$ vanishes identically for $l_{2}>\frac{1}{2}$. Similarly we can show that $l_{n} \leq 1 / n$ for all $n$ and $p_{n}\left(l_{n}\right)=0$ for $l_{n}>1 / n$.

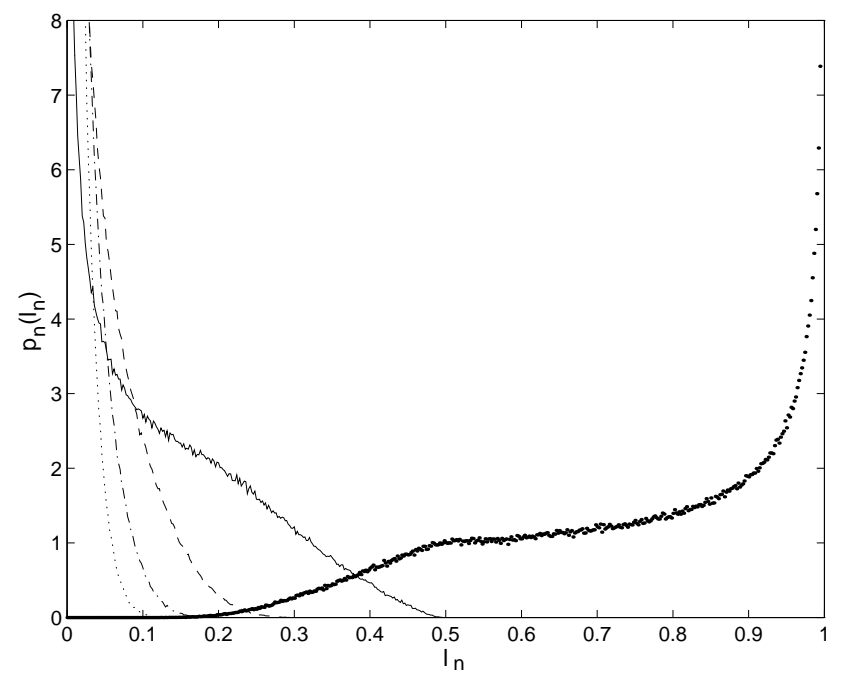

FIG. 2. Probability densities of the 5 longest loops from right to left: $p_{1}\left(l_{1}\right)$-thick points, $p_{2}\left(l_{2}\right)$-solid line, $p_{3}\left(l_{3}\right)$-dashed line, $p_{4}\left(l_{4}\right)$-dot-dashed line, $p_{5}\left(l_{5}\right)$-dotted line, as a function of $l_{n}=L_{n} / N$ from MC results of $10^{6}$ random sequences of length $N=1000$.

We note that $p_{2}\left(l_{2}\right)$ is qualitatively similar to the probability densities of the length of the second segment in different models of randomly broken objects [26,27] (see Figs. 1(b) and 3(b) in [26]). However, the probability densities of the length of the second segment in [26] have strong singularities (of the first derivative) at $l_{2}=1 / 4$, and are shown to have singularities at $l_{2}=1 / k$ for all integer $k \geq 2$. All the probability densities $p_{n}\left(l_{n}\right)$ of the longest loops have a singularity when they become identically zero $\left(p_{n}\left(l_{n}\right)\right.$ vanishes for $l_{n}>1 / n$ and therefore is non-analytical at $\left.l_{n}=1 / n\right)$. It is possible that due to the singularity in $p_{n^{\prime}}\left(l_{n^{\prime}}\right)$ at $1 / n^{\prime}$ all the other probability densities $p_{n}\left(l_{n}\right)$ (for $n<n^{\prime}$ ) also have singularities at $1 / n^{\prime}$, since all the probability densities are dependent. Apparently, these singularities do not cause discontinuity of the first derivative, and, therefore are not visible in the numeric data. In several models of randomly broken objects 26, 27, the probability densities of the length of the $n$th segment have singularities at $l=1 / n^{\prime}$ (for $n \leq n^{\prime}$ ). These singularities are due to a self-similar process, which leads to a different analytical expression for the probabilities on each interval $1 /\left(n^{\prime}+1\right)<l<1 / n^{\prime}$. Since the random process of generating the longest loops in our process is not self-similar (as indicated in the previous section), the reason for the singularities in the models of randomly broken objects does not hold in the case of the $n$th longest loop (as was suggested by Frachebourg et al. [29]). Therefore, the probability of the $n$th longest loop does not necessarily have singularities at values of $l=1 / n^{\prime}$.

Since in any specific RW, $L_{1} \geq L_{2} \geq \cdots \geq L_{n}$, the average length of the $n$th longest loop $\left\langle L_{n}\right\rangle$ decreases (for fixed $N$ ) with increasing loop number $n$. There is no typical scale in the problem, and we may expect a power law dependence $\left\langle L_{n}\right\rangle \sim N n^{-\alpha}$, with $\alpha>0$. The total number of steps in all the loops in any given RW cannot exceed $N$ (i.e. $\sum_{n} L_{n} \leq N$ ), and therefore $\sum_{n}\left\langle L_{n}\right\rangle \leq N$. The convergence of the sum means that $\alpha>1$. We depict in Fig. 3 the average reduced length $\left\langle l_{n}\right\rangle$ vs. $n$ on a logarithmic scale. To avoid systematic errors due to finite $N$, each value of $\left\langle l_{n}\right\rangle$ in the graph was determined through an extrapolation: For each $n$, we plotted $\left\langle l_{n}\right\rangle$ vs. $1 / N$, and found the extrapolated value of $\left\langle l_{n}\right\rangle$ near $1 / N=0$. These values of $\left\langle l_{n}\right\rangle$ are depicted in Fig. 3. The linear fit to the data points has a slope of $-2.3 \pm 0.4$. We therefore conclude that as $N \rightarrow \infty$ :

$$
\left\langle L_{n}\right\rangle \sim \frac{N}{n^{\alpha}}, \text { where } \alpha=2.3 \pm 0.4
$$

At the next section we will argue that $\alpha=2$, which is within the error limits of Eq. (3).

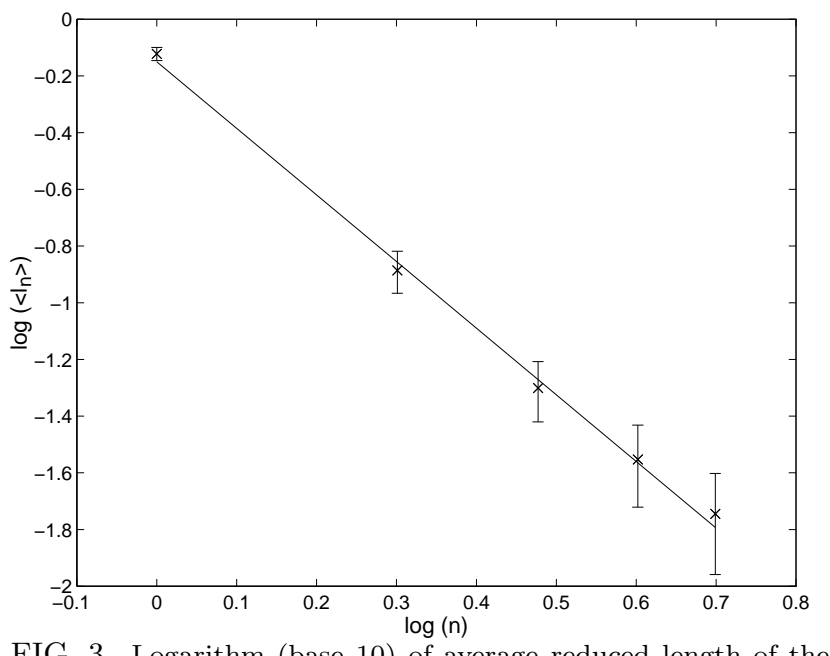

FIG. 3. Logarithm (base 10) of average reduced length of the $n$th longest loop as a function of logarithm of loop number $n$. 


\section{NEUTRAL SEGMENTS IN FINITE CHAINS}

So far we have discussed only long chains and their properties in the $N \rightarrow \infty$ limit. There are several differences between the properties of infinitely long chains and finite size chains. First of all, in finite size chains the monomers that do not belong to any neutral segment constitute a finite part of the chain, as opposed to a vanishing part as $N \rightarrow \infty$. Secondly, in infinitely long chains the number of neutral segments is infinite, while for finite $N$ at some point there are no more neutral segments.

Let us consider the total number of monomers which do not belong to any neutral segment, for finite $N$. It is easily shown that the number of monomers left out from all the neutral segments in any specific random sequence is exactly the excess charge $Q$ of the corresponding PA: The excess charge cannot exceed the number of monomers left out, because, by definition, the neutral segments do not have excess charge. On the other hand, all the monomers left out from the neutral segments must be part of the excess charge: If, for instance, a PA has excess positive charge, then no negative charge can be left out from all the neutral segments. (If there had been a group of negative charges, it would have joined a group of positive charges to make a neutral segment, or together with a group of positive charges, would have joined an existing neutral segment and make it longer). Since the r.m.s. excess charge of a randomly charged sequence of $N$ charges of \pm 1 is equal to $\sqrt{N}$, the r.m.s. number of monomers not in any neutral segment in the chain is also equal to $\sqrt{N}$, becoming a vanishing fraction of the chain as $N \rightarrow \infty$.

At some point the process of search for the next longest loop exhausts all the loops in the RW. We investigate this stage in the process, by analyzing the number $n_{f}$ of loops in a RW. When $N$ is infinite, the average length of the $n$th longest loop is given by $\left\langle L_{n}\right\rangle \sim N n^{-\alpha}$. Application of this equality for finite $N$ 's would predict, for large enough $n$ 's, $\left\langle L_{n}\right\rangle \leq 1$. Since this is not possible (the minimal length of a loop is two steps), we argue that Eq. (3) is valid, for finite $N$ 's, only to describe the average lengths of the longest $n_{f}$ loops. There is no typical scale to the problem of the total number of loops, and we therefore expect a power law dependence of $\left\langle n_{f}\right\rangle$ on $N$. The length of the last loop, for all chain lengths, is usually very short (consisting of only few positive and negative charges), having length independent of $N$, i.e. $\left\langle L_{n_{f}}\right\rangle \sim N^{0}$, which means that $\left\langle l_{n_{f}}\right\rangle \sim N^{-1}$. Since $\left\langle l_{n_{f}}\right\rangle \sim n_{f}^{-\alpha}$ (substituting $n=n_{f}$ in Eq. 3), we can expect:

$$
\left\langle n_{f}\right\rangle \sim N^{y}, \quad \text { where } y=\frac{1}{\alpha} .
$$

Substituting $\alpha$ from Eq. (3), leads to a value of $y=$ $0.43 \pm 0.09$. Investigating numerically the dependence of $\left\langle n_{f}\right\rangle$ on $N$, we found that $y=0.46 \pm 0.06$, which is within the error limits of the value predicted by Eqs. (3) and (4).
At the end of this section we will argue that $\alpha=2$ and $y=0.5$, values which are within the error limits of those deduced from the numeric data. In order to confirm the $\left\langle n_{f}\right\rangle \sim \sqrt{N}$ relation, we show in Fig. 1 the probability density of $n_{f}$ divided by $\sqrt{N}$ for several chain lengths $\left(N=10^{2} \div 10^{4}\right)$. The division by $\sqrt{N}$ causes a reasonable collapse of the graphs for different values of $N$ to a single function, which is (almost) $N$-independent. We see that the probability density has a maximum when $n_{f} / \sqrt{N}$ is close to zero (the most probable value of $n_{f}$ is finite and independent of $N$ ), and it decreases to zero with increasing $n_{f} / \sqrt{N}$.

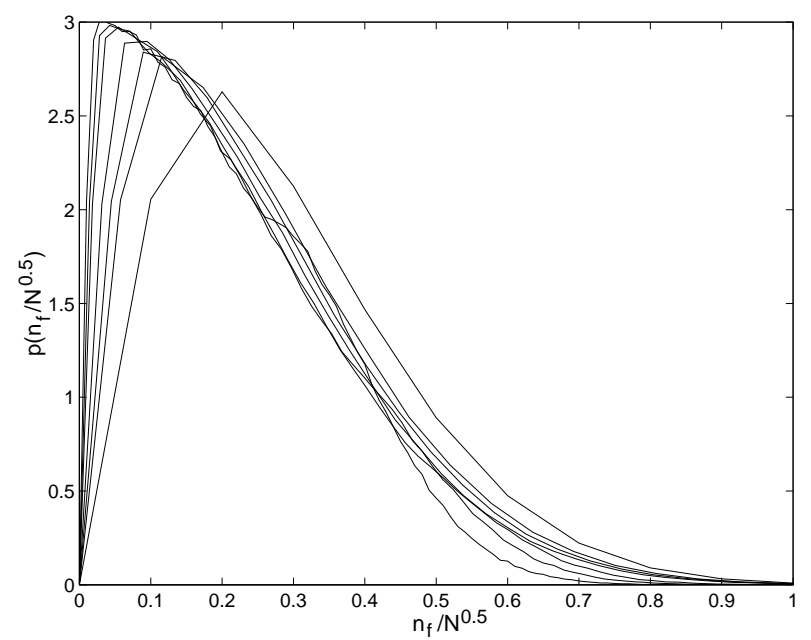

FIG. 4. Probability density of $n_{f}$, the total number of loops in a chain, divided by $\sqrt{N}$, for $N=100,300,500,1000,3000,5000$, 10000 (from right to left).

Knowing the statistical properties of the chain, we try to construct a self-consistent complete picture, in which our numerical results fit together. We know that the total length of all the loops $\left(\sum_{n=1}^{n_{f}} L_{n}\right)$ equals to the entire length of the chain minus the steps not in any loop (which are the excess charge, which are on the average $\sqrt{N})$. Dividing this equality by $N$, taking an average over the random sequences, we get:

$$
1-\frac{1}{N^{\frac{1}{2}}}=\left\langle\sum_{n=1}^{n_{f}} l_{n}\right\rangle
$$

On the other hand, from Eqs. (3) and (4) we know that, omitting constants of order unity:

$$
\left\langle\sum_{n=1}^{\left\langle n_{f}\right\rangle} l_{n}\right\rangle=\sum_{n=1}^{\left\langle n_{f}\right\rangle}\left\langle l_{n}\right\rangle \simeq \int_{n=1}^{N^{y}} n^{-\alpha} d n \sim 1-N^{y(1-\alpha)} .
$$

Comparison of the powers of $N$ in Eqs. (5) and (6) leads to

$$
y=\frac{1}{2(\alpha-1)}
$$


which together with Eq. (4) is satisfied by:

$$
\alpha=2, \quad y=\frac{1}{2} .
$$

These equalities are satisfied by the values of $y=0.46 \pm$ 0.06 , and $\alpha=2.3 \pm 0.4$ obtained numerically, and constitute a self-consistent picture, in which the average conformational properties of the constructed ground state fit together.

\section{PHYSICAL PROPERTIES OF THE GROUND STATE}

In this section we investigate some of the physical characteristics of the constructed ground state of randomly charged PA's. We focus on the linear size $R$ and on the surface area $S$ of the proposed ground state, trying to explain their dependencies on $N$ through the self consistent picture constructed in the previous section. We define $R$, the linear size of the chain, according to the picture of Fig. i: The neutral segments in the chain compact into globules (each with a linear size of $R_{\text {segment }} \sim L_{\text {segment }}^{1 / 3}$ ). If all the globules are linearly packed then the total linear size is the sum of the linear sizes of all the globules $\left(R=\sum R_{\text {segment }}\right)$. The linear size of the chain must include the monomers not in any neutral segment (the 'strings' in the necklace, which are the total excess charge $Q)$. We therefore get a means to describe the chain's size:

$$
R \equiv \sum_{n=1}^{n_{f}} L_{n}^{1 / 3}+Q
$$

(Here, and throughout this section, we omit prefactors of order unity.) It is evident that the generated state captures some essential features of the ground state suggested by the necklace model: The necklace type structure is compact (i.e. $R \sim N^{1 / 3}$ ) when the PA is neutral (the longest neutral segment is the entire chain) or has very small excess charge, and begins to stretch as the excess charge increases (the charged strings become longer). Finally, the PA becomes completely stretched (i.e. $R \sim N$ ) for the fully charged polymer.

Investigating the average chain's linear size dependence on $N$, we get the dependence:

$$
\langle R\rangle \sim N^{\nu}, \text { where } \nu=0.50 \pm 0.01 .
$$

Fig. . $\sqrt{N}$ for several values of $N$. From the data collapse we deduce that the $N$-dependence in Eq. (10) is valid not just for the average linear size, but represents a scaling of the entire probability density. The $\nu \simeq \frac{1}{2}$ power in Eq. (10) means that the chain is not compact (although the distribution is 'peaked' near the lowest possible value of $R$ ), and is not completely stretched, but has a linear size as an ideal RW with $N$ steps.

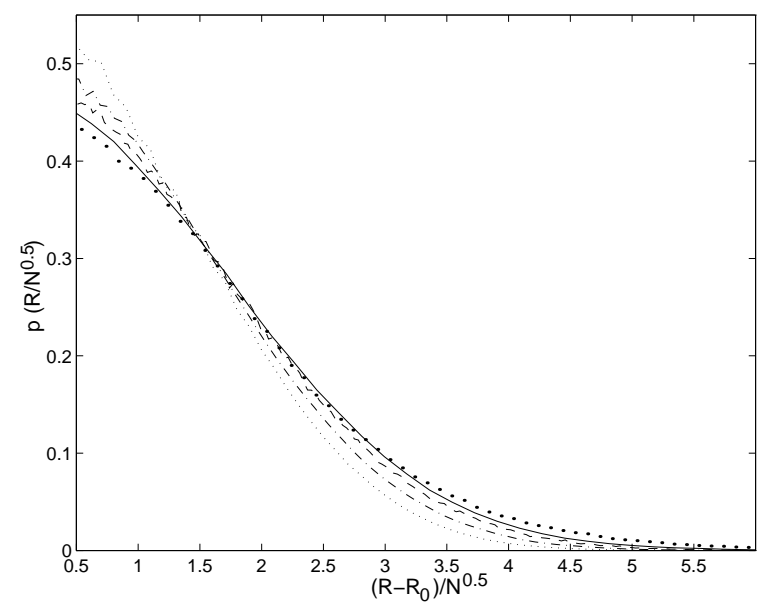

FIG. 5. Probability density of $R$, the linear size of the entire chain, for several chain lengths: $N=100$ (dotted line), 300 (dot-dashed line), 1000 (dashed line), 3000 (solid line), 10000 (thick points). The minimal possible value of $R\left(R_{0}=N^{1 / 3}\right)$ is subtracted from $R$, and the result is divided by $\sqrt{N}$ to collapse the data.

We can explain the dependence in Eq. (10), by assuming that $\sum_{n}\left\langle L_{n}{ }^{1 / 3}\right\rangle$ and $\sum_{n}\left\langle L_{n}\right\rangle^{1 / 3}$ have the same $N$-dependence, and by using the power laws of $\left\langle n_{f}\right\rangle$ and $\left\langle L_{n}\right\rangle$ (Eqs. 3 and 1 ), with $\alpha=2$ and $y=\frac{1}{2}$ (Eq. 8):

$$
\sum_{n=1}^{n_{f}}\left\langle L_{n}^{1 / 3}\right\rangle \sim \sum_{n=1}^{\left\langle n_{f}\right\rangle}\left\langle L_{n}\right\rangle^{1 / 3} \sim \int_{n=1}^{N^{y}}\left(\frac{N}{n^{\alpha}}\right)^{1 / 3} \sim N^{0.5} .
$$

In order to confirm this dependence, we investigated $\left\langle\sum_{n=1}^{n_{f}} L_{n}^{1 / 3}\right\rangle$ as a function of $N$, and found the power law dependence with exponent $0.49 \pm 0.02$, in accordance with the prediction of Eq. (11). We see that the average linear chain size $\langle R\rangle$ in Eq. (9) is a sum of two terms, each proportional to $\sqrt{N}$.

Similarly to the definition of $R$, we can define the surface area $S$ of the chain. Each neutral segment compacts into a globule of surface area $\sim L_{\text {segment }}^{2 / 3}$, all the globules are linearly packed, and the surface area of the 'strings' is proportional to the number of monomers not in any neutral segment, which is the excess charge. We can therefore define:

$$
S \equiv \sum_{n=1}^{n_{f}} L_{n}^{2 / 3}+Q
$$

$S$ can have values ranging from $N^{2 / 3}$, for a neutral chain, to $N$, for a completely charged chain. These limits indeed correspond to the expected behavior of PA. Obviously $S \geq N^{2 / 3}$. We analyzed the $N$-dependence of $S$, and found that:

$$
\langle S\rangle \sim N^{0.67 \pm 0.01}
$$


When we subtract from $S$ its minimal value, and divide the result by $N^{2 / 3}$, we get a distribution which is identical for all $N$. This dependence means that the average surface energy of the generated structure has the same $N$-dependence as the surface energy of a single compact globule (or several compact globules, each containing a finite part of the chain). From the same arguments that led to Eq. (11), we get:

$$
\langle S\rangle=\langle Q\rangle+\sum_{n=1}^{\left\langle n_{f}\right\rangle}\left\langle L_{n}\right\rangle^{2 / 3} \sim \sqrt{N}+\int_{n=1}^{N^{y}}\left(\frac{N}{n^{\alpha}}\right)^{2 / 3} \sim N^{2 / 3} .
$$

This power of $N$ is in accordance with the numerically obtained value of $0.67 \pm 0.01$, and indicates that the $N$ dependence of the average surface area is determined by the neutral segments (i.e. the 'beads' in the necklace), and is not affected by the excess charge (the 'strings' in the necklace).

We investigated the expression $S+\frac{Q^{2}}{R}$, which has the same $N$-dependence as the energy of the generated structure. (We considered the energy terms of Eq. (11), and omitted the condensation term, since it is the same for all structures of a given $N)$. Exploring the $N$-dependence of $S+\frac{Q^{2}}{R}$ (denoted as $E$ ), we found that:

$$
\langle E\rangle \equiv\left\langle S+\frac{Q^{2}}{R}\right\rangle \sim N^{0.66 \pm 0.01} .
$$

When subtracting from $E$ its minimal value and dividing the result by $N^{2 / 3}$, we get a probability density which is identical for all $N$. This dependence means that the total energy is very low: The surface energy term $(S)$ gets its minimal value $\left(\sim N^{2 / 3}\right)$, and $R \sim N^{1 / 2}$, thus bringing the electrostatic energy term to a point where it does not affect the $N$-dependence of the total energy. The total energy behaves very much like the surface energy, as if the chain is constructed of a single compact neutral globule.

\section{CONCLUSIONS AND DISCUSSION}

We have studied the properties of a conjectured structure of randomly charged PA's in the ground state. According to the necklace model 13,14, in the ground state of PA's neutral segments in the chain compact into globules. Following Kantor and Ertaş [17, 18, we have mapped the problem of longest neutral segments to the problem of longest loops in 1-d RW's, and applied numerical methods along with analytical estimates to study the size distribution of longest loops.

Since we believe that a structure of PA's based on the necklace model has a very low energy, we suggested a specific detailed necklace-type structure for polyampholytes in the ground state, and numerically obtained its conformational and physical properties (the number and sizes of 'beads' and 'strings' in the necklace, the spatial extent, the surface area and energy). This structure is compact when the chain is neutral or weakly charged, and stretches as the chain becomes charged. We have found that the ground state structure has a very low energy, which depends on the number of monomers as the energy of a single compact neutral globule. We have also shown that the unrestricted average of the linear size of the polymer in the ground state depends on the number of monomers as the linear size of an ideal chain, with a critical exponent of $\nu=0.50 \pm 0.01$. Although the qualitative behavior of our distribution for the linear size is similar to this obtained in [15] (it is peaked near its smallest possible value, and has a tail, which determines the asymptotic behavior of $\nu$ ), Kantor and Kardar 15] concluded that the average linear size increases with $N$ at least as fast as a self-avoiding walk (i.e. $\nu>0.6$ ). We believe that it is worthwhile to slightly alter the way in which compact globules are formed within our model (by allowing for weakly charged globules or by not forcing all the neutral segments to completely compact), in order to try to reproduce this 'swelling' of the average chain.

The investigation of the size distribution of neutral segments through the analogy to 1-d RW's, was limited to a particular class of RW's, in which a unit displacement appears at each step (i.e. each monomer in the chain is charged \pm 1 ). In a future publication 28, we define the problem of longest loops in the limit where the RW becomes a true Gaussian walk, and prove through a scaling process the universality of the probability densities of longest loops. This universality means that the probability densities of longest loops are independent of the number of steps and of the nature of the single step of the RW. Therefore, the results obtained for a specific case of randomly charged PA's are valid for all randomly charged polymers, in which (for long chains) the charge distribution is an unbiased Gaussian.

\section{ACKNOWLEDGMENTS}

This work was supported by the Israel Science Foundation under grant No. 246/96.

[1] T. E. Creighton, Proteins: Structures and Molecular Properties, 2nd ed. (W. H. Freeman and Company, New York, 1993).

[2] P. G. de Gennes, Scaling Concepts in Polymer Physics (Cornell University Press, Ithaca, New York, 1979).

[3] A. Y. Grosberg and A. R. Khokhlov, Statistical Physics of Macromolecules (AIP Press, New York, 1994).

[4] C. Tanford, Physical Chemistry of Macromolecules (John Wiley and Sons, New York, 1961). 
[5] P. G. Higgs and J. F. Joanny, J. Chem. Phys. 94, 1543 (1991).

[6] J. Wittmer, A. Johner and J. F. Joanny, Europhys. Lett. 24, 263 (1993).

[7] S. F. Edwards, P. R. King and P. Pincus, Ferroelectrics 30, 3 (1980).

[8] L. D. Landau and E. M. Lifshitz, Statistical Physics (Pergamon Press, New York, 1980).

[9] Y. Kantor and M. Kardar, Europhys. Lett. 14, 421 (1991).

[10] Y. Kantor, H. Li and M. Kardar, Phys. Rev. Lett. 69, 61 (1992).

[11] Y. Kantor, M. Kardar and H. Li, Phys. Rev. E 49, 1383 (1994).

[12] D. Bratko and A. M. Chakraborty, J. Phys. Chem. 100, 1164 (1996).

[13] Y. Kantor and M. Kardar, Europhys. Lett. 27, 643 (1994).

[14] Y. Kantor and M. Kardar, Phys. Rev. E 51, 1299 (1995).

[15] Y. Kantor and M. Kardar, Phys. Rev. E 52, 835 (1995).
[16] A. V. Dobrynin, M. Rubinstein and S. P. Obukhov, Macromolecules 29, 2974 (1996).

[17] D. Ertaş and Y. Kantor, J. Phys. A27, L907 (1994).

[18] Y. Kantor and D. Ertaş, Phys. Rev. E 53, 846 (1996).

[19] D. Ertaş and Y. Kantor, Phys. Rev. E 55, 261 (1997).

[20] G. F. Lawler, Duke Math. J. 47, 655 (1980).

[21] G. F. Lawler, Duke Math. J. 53, 249 (1986).

[22] G. F. Lawler, J. Stat. Phys. 50, 91 (1988).

[23] A. J. Guttmann and R. J. Bursill, J. Stat. Phys. 59, 1 (1990).

[24] R. E. Bradley and S. Windwer, Phys. Rev. E 51, 241 (1995).

[25] D. Dhar and A. Dhar, Phys. Rev. E 55, 2093 (1997).

[26] B. Derrida and H. Flyvbjerg, J. Phys. A20, 5273 (1987).

[27] B. Derrida and H. Flyvbjerg, J. Phys. A19, L1003 (1986).

[28] S. Wolfling and Y. Kantor (to be published).

[29] L. Frachebourg, I. Ispolatov and P. L. Krapivsky, Phys. Rev. E 52, R5727 (1995). 